\title{
Concept for the Keck Next Generation Adaptive Optics system
}

Donald Gavel, Richard Dekany, Claire Max, Peter Wizinowich, Sean Adkins, et al.

Donald Gavel, Richard Dekany, Claire Max, Peter Wizinowich, Sean Adkins, Brian Bauman, Jim Bell, Erik Johansson, Renate Kupke, Chris Lockwood, Anna Moore, Chris Neyman, Marc Reinig, Viswa Velur, "Concept for the Keck Next Generation Adaptive Optics system," Proc. SPIE 7015, Adaptive Optics Systems, 701567 (15 July 2008); doi: $10.1117 / 12.790250$

Event: SPIE Astronomical Telescopes + Instrumentation, 2008, Marseille, France 


\title{
Concept for the Keck Next Generation Adaptive Optics System
}

\author{
Donald. Gavel $^{* a}$, Richard Dekany ${ }^{\mathrm{c}}$, Claire Max ${ }^{\mathrm{a}}$, Peter Wizinowich ${ }^{\mathrm{b}}$, Sean Adkins ${ }^{\mathrm{b}}$, Brian Bauman ${ }^{\mathrm{d}}$, \\ Jim Bell $^{\mathrm{b}}$, Erik Johansson ${ }^{\mathrm{b}}$, Renate Kupke ${ }^{\mathrm{a}}$, Chris Lockwood ${ }^{\mathrm{a}}$, Anna Moore ${ }^{\mathrm{c}}$, Chris Neyman ${ }^{\mathrm{b}}$, Marc \\ Reinig $^{\mathrm{a}}$, Viswa Velur ${ }^{\mathrm{c}}$ \\ ${ }^{a}$ UCO Lick Observatory, UC Santa Cruz, 1156 High Street, Santa Cruz, CA, USA 95060; ${ }^{b}$ Keck \\ Observatory, 65-1120 Mamalahoa Hwy, Waimea, HI, 96796; ${ }^{\circ}$ Caltech Optical Observatories,

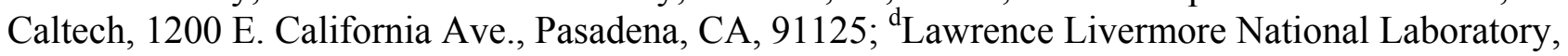 \\ 800 East Ave., Livermore, CA 94550
}

\begin{abstract}
The Next Generation Adaptive Optics (NGAO) system will represent a considerable advancement for high resolution astronomical imaging and spectroscopy at the W. M. Keck Observatory. The AO system will incorporate multiple laser guidestar tomography to increase the corrected field of view and remove the cone effect inherent to single laser guide star systems. The improvement will permit higher Strehl correction in the near-infrared and diffraction-limited correction down to R band. A high actuator count micro-electromechanical system (MEMS) deformable mirror will provide the on-axis wavefront correction to a number of instrument stations and additional MEMS devices will feed multiple channels of a deployable integral-field spectrograph. In this paper we present the status of the AO system design and describe its various operating modes.
\end{abstract}

Keywords: Adaptive Optics, Keck, MEMS, Tomography

\section{INTRODUCTION}

The Keck Laser Guidestar Adaptive Optics system is one of the pioneer LGS AO systems for use in astronomy and, since it's commissioning in 2004, has produced breakthrough results in high resolution imaging and spectroscopy.[1] In consideration of the strategic positioning of the W. M. Keck Observatory in the era of large telescopes, and in view of the recent advances in adaptive optics technology, the Observatory has commissioned a design study for a Next Generation Adaptive Optics (NGAO) system. The NGAO system will incorporate multiple laser guidestar tomography in order to increase the field of view and wavelength range available for diffraction-limited imaging, thus enhancing the science reach of the telescope. The overall facility description and science cases are provided in companion papers at this conference.[2][3] In this paper we describe the AO system baseline opto-mechanical design, highlighting the capabilities and tradeoffs.

A key feature of the KNGAO baseline system design is its novel architecture and targeted use of new technology. The system uses multiple laser guide star wavefront sensing and a dedicated real-time processor to do atmospheric tomography. The AO system architecture consists of a "cascaded," woofer-tweeter design with a conventional DM (woofer) in the first relay, and a high actuator count micro-electromechanical system (MEMS) deformable mirror in the second stage. The MEMS DM, a 4096 actuator device under development initially for application in the Gemini Planet Finder[5], will provide high Strehl on-axis wavefront correction.

KNGAO will also provide a wide-field multi-object science capability, with the baseline design being 6 deployable integral field spectrographs, located in the field after the first relay. Each deployable arm has its own, 1024 actuator MEMS, deformable mirror. In support of laser guide star wavefront sensing, two or three natural stars must be selected over a wide field for tip/tilt and focus sensing. These will also be corrected by AO through the use of 1024 actuator MEMS and sensed in the infrared. The AO sharpening of tip/tilt stars allows much dimmer stars to be used, which dramatically improves the fractional sky coverage and performance in LGS AO observing mode.[6]

*gavel@ucolick.org; phone 1831 459-5464

Adaptive Optics Systems, edited by Norbert Hubin, Claire E. Max, Peter L. Wizinowich,

Proc. of SPIE Vol. 7015, 701567, (2008)

0277-786X/08/\$18 doi: $10.1117 / 12.790250$

Proc. of SPIE Vol. 7015 701567-1 
In addition to LGS AO capability, the system will be operational in natural guide star (NGS) mode. A selectable sampling on the NGS wavefront sensor allows for a range of natural guide star brightness.

\section{OPTOMECHANICAL RELAY}

\subsection{Requirements}

The design of the NGAO relay was driven by requirements derived from the science objectives of the NGAO. These include, but are not limited to:

- Location

- The AO system will reside on the Nasmyth Platform

- Field and Pupil Rotation

- The optical relay will have a K-mirror upstream of the science instruments, wavefronts sensors, and tip-tilt sensors.

- Field of View

- A high Strehl science relay with a field of view (FOV) of between 20" and 40" diameter.

- A 150" diameter FOV interfacing to a deployable IFU device.

- A 175" diameter FOV at the laser guide star (LGS) focus, feeding the LGS high-order wavefront sensors (HOWFS).

- A $150^{\prime \prime}$ diameter FOV at the natural guide star (NGS) focus, providing NGS for tip-tilt, and low-order wavefront sensing (LOWFS)

- Pupil Diameter

- The first relay shall contain a low-order "woofer" mirror, conjugate to the telescope primary, with a diameter of $100 \mathrm{~mm}$.

- The second relay shall contain a high-order, MEMs "tweeter" mirror, conjugate to the telescope primary, with a diameter of $25 \mathrm{~mm}$.

- Output Pupil Location

- The exit pupil for the first relay will be telecentric to simplify design of the pick-off mechanisms and wavefront sensors for the LOWFS and HOWFS.

- Output Focal Relays

- The output focal ratio of the first relay shall match the focal ratio of the $f / 15$ telescope Nasmyth focus.

- The output focal ratio of the second relay shall be greater than $\mathrm{f} / 45$ to allow for the optical switchyard.

\subsection{Architecture of the Design}

The proposed Keck NGAO architecture consists basically of two pupil relays in cascade, a wide field relay followed by a narrow field one. The wide field relay contains a "woofer" deformable mirror (nominally 20 actuators across the aperture) conjugate to the ground layer. After the DM, light from the constellation of laser guide stars is split by a dichroic and sent to a wavefront sensor array. The wavefront sensor signals are processed by a real-time tomography computer which continuously estimates the delta-index variations in the volume of atmosphere above the telescope. Science light continues on to either be fed to a wide field deployable integral field spectrometer (dIFS), or into the narrow field relay. Science objects for the dIFS are selected with pickoff arms which send small patches of the field into a spectrometer. Along this path, a second "tweeter" DM, a MEMS device, further corrects the wavefront specific to this particular science direction using information from the tomography computer. This configuration, known as MultiObject AO (MOAO) permits correction of the anisoplanatic component of the wavefronts from the off-axis science objects.

The narrow field relay contains a second "tweeter" deformable mirror, nominally 64 actuators across and also conjugate to the ground, which can feed high resolution narrow-field instruments, such as a near infrared or visible spectrometer and imagers.

Tip/tilt stars are selected from a field split from the output of the wide field relay and fed to a tip/tilt sensor. In order to accommodate fluctuations in mean height of the sodium layer, one of these sensors will also be able to sense focus, hence as a group they are referred to as the Low-Order Wavefront Sensors (LOWFS). The tip/tilt star pickoff mechanism might be to that used by the dIFS. Selecting tip/tilt/focus stars from the wide field gives the maximum flexibility in 
finding up to 3 tip/tilt guide stars needed to fill in the blind modes unsensed by the laser guidestars (due to up-path tip/tilt uncertainty). The tip/tilt arms will each themselves contain in their optical path a MEMS deformable mirror, nominally 32 actuators across, which sharpens the star to the diffraction limit. This increases the sensitivity of the tip/tilt sensor allowing dimmer tip/tilt stars to be usable and thereby significantly increasing LGS AO sky coverage.

All of the MEMS deformable mirrors (the tweeter in the second relay, the DMs in the dIFS, and the DMs in the tip/tilt/focus sensors) will be operated in "Multi-object AO" (MOAO) configuration. In this mode, guide star light is not reflected off of the DMs and hence they must be run open-loop. Experiments at Lick Observatory have demonstrated that the electrostatic DMs baselined for KNGAO are capable of being operated open loop and still perform accurate wavefront reconstruction.[7][8]

For natural guide star operation, a single natural guidestar wavefront sensor is located at the output of the narrow field $\mathrm{AO}$ relay. This is also the location of the truth wavefront sensor used for slow calibration wavefront sensing in laser guide star mode.

The AO system will be mounted on the Nasmyth platform of the Keck Alt-Az telescope. Field derotation is accomplished by a single wide-field K-mirror mechanism, located ahead of the AO system's first relay near the telescope focus.

\subsection{Opto-mechanical Layout}

An annotated optical layout of the relay can be seen in Figure 1, alongside a Solidworks drawing of the mechanical structures needed to support and move the optics, as well as the locations of wavefront sensors and instruments, in Figure 2 .

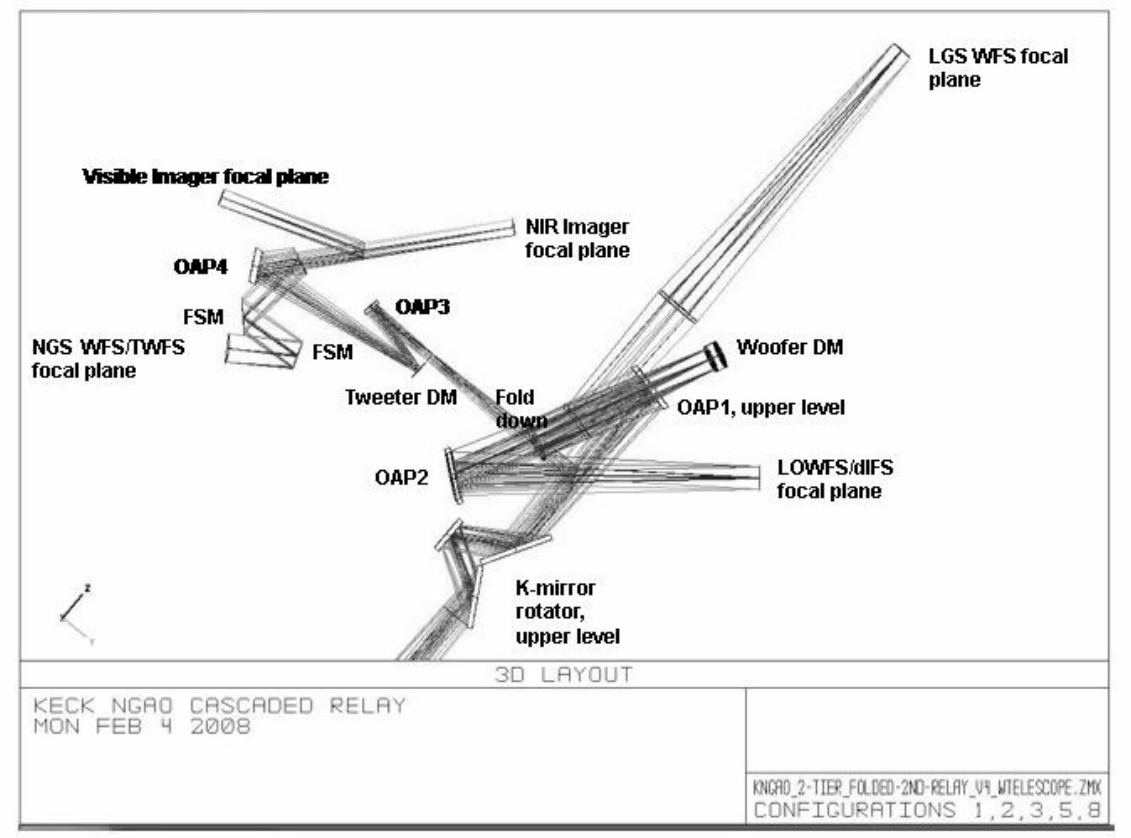

Figure 1. Optical layout, Cascaded Relay

Several optical designs were attempted in order to produce un-vignetted light paths, acceptable locations for focal planes, and clearance for supports and mechanisms. The $1^{\text {st }}$ relay of the two-relay NGAO system is similar to the existing Keck AO system. And probably in a similar evolution, it became apparent that without a fold in the $1^{\text {st }}$ relay of the new system the various foci would end up in difficult and in some cases impossible positions.

This fold mirror along with the low-order deformable mirror allow for a periscope type split level relay. Solutions were explored for both in-plane, and split-level (or two-tiered) solutions. The least difficult configuration to package was a two-tier design shown below in Figure 3. 
The two-tier $1^{\text {st }}$ relay requires a platform elevated above the level of the main optical table. Three components are suspended from the under side of this platform: OAP1; flat fold mirror; and the image rotator (K-mirror). The upper and lower tiers are expected to be typical honeycomb core optical tables of custom plan form. Thickness determination will occur is subsequent design phases. The separation of these two is accomplished with three bi-pod struts. If necessary, there is clearance to make these tripod struts, allowing kinematic mount detail for the upper tier.

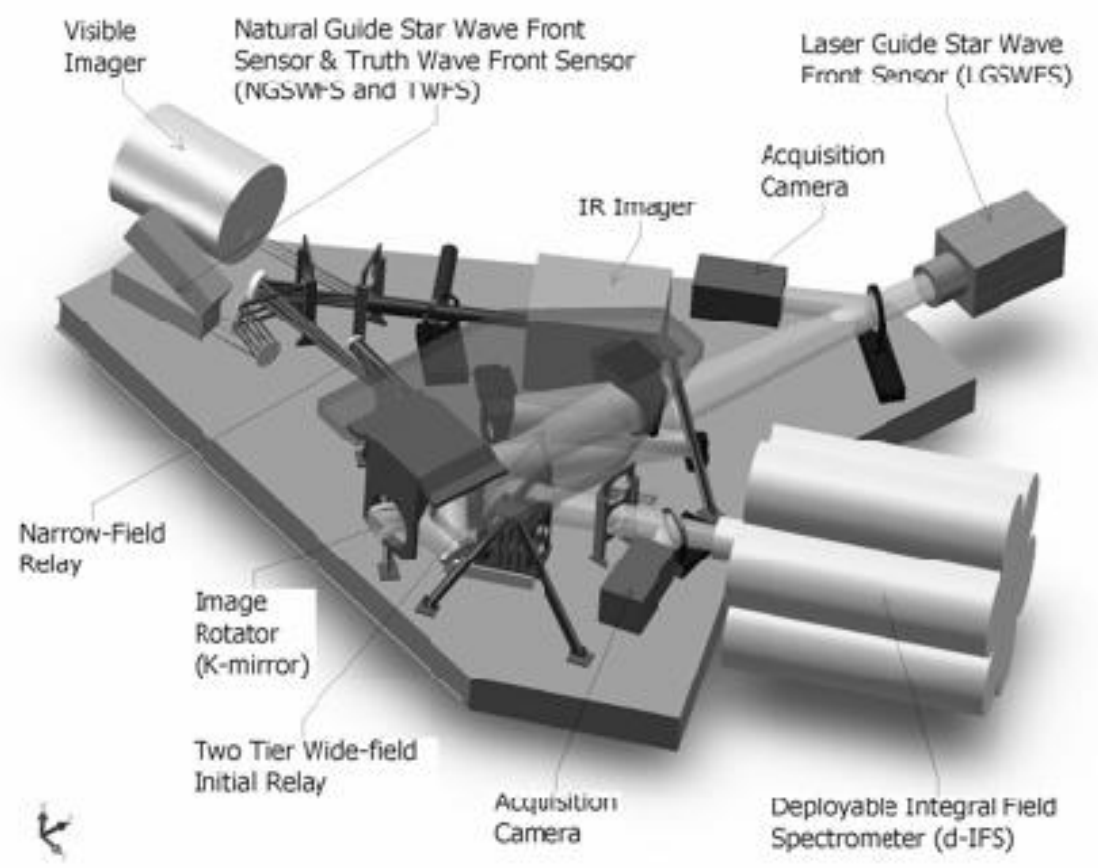

Figure 2. Overall layout, Cascaded Relay

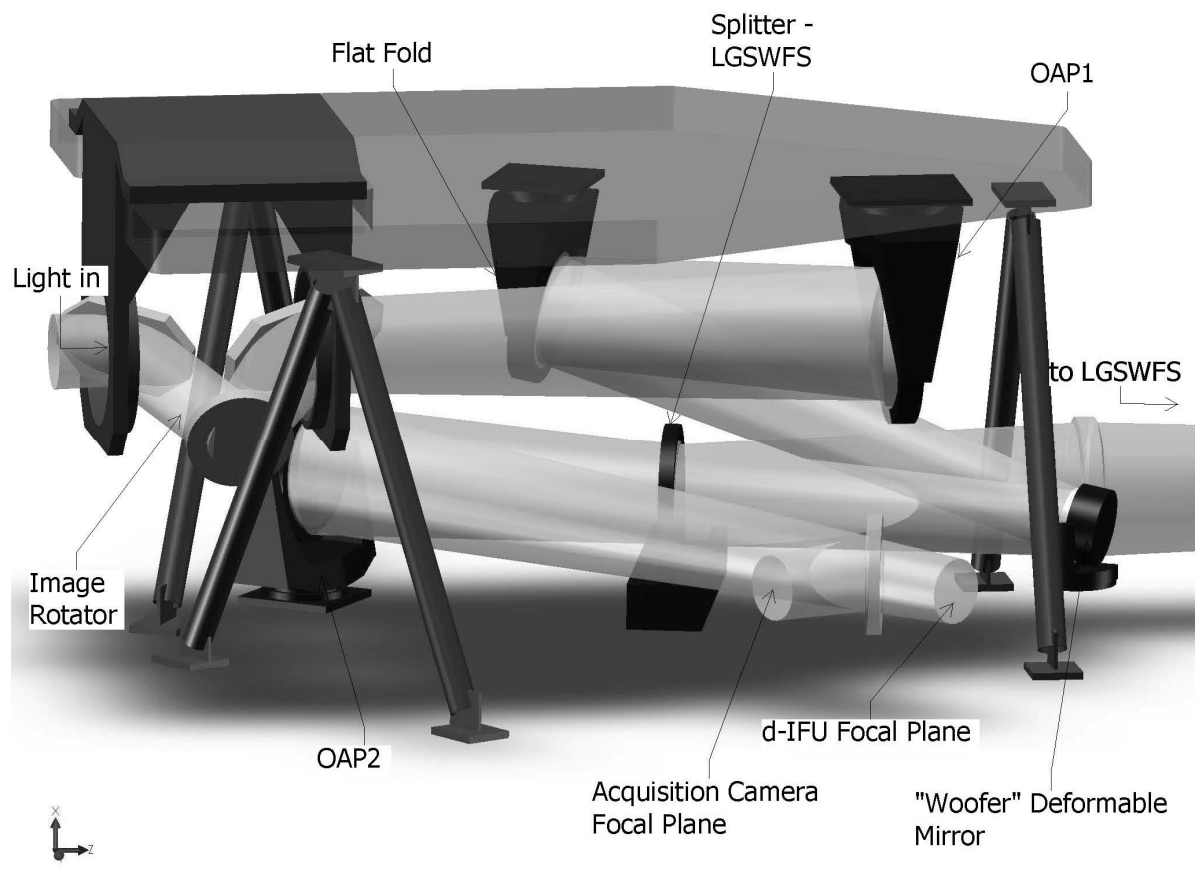

Figure 3. $1^{\text {st }}$ Relay - Isolated. This figure shows the elements of the Two-Tiered $1^{\text {st }}$ relay. Light enters from the telescope at left, travels through the rotator to OAP1, to the flat fold, to the Woofer DM at the pupil. It proceeds to a dichroic that 
splits light to the LGSWFS and OAP2. From OAP2 it travels to the d-IFU focus. A (retractable) mirror is also shown just prior to this focus to steer the light into an Acquisition Camera for NGS mode.

\subsection{Optical Performance 1 - Focal Plane Wavefront Quality}

The optical performance of the NGAO relays is excellent in both the wide and narrow field science legs, and in the path to the LOWFS. This minimizes the use of the DMs in correcting for internal optical aberarions.

Table 1. Performance of the dIFS relay.

\begin{tabular}{|c|c|c|c|c|c|c|c|c|}
\hline Instrument & $\lambda$ bands & F/\# & FOV (") & $\begin{array}{c}\text { Field } \\
\text { Curvature } \\
(\mathbf{m m})\end{array}$ & $\begin{array}{c}\text { RMS } \\
\text { WFE }(\mathbf{n m})\end{array}$ & $\begin{array}{c}\text { Spot Size } \\
(\mu) \text { at J }\end{array}$ & $\begin{array}{c}\text { Spot size } \\
\left(\mathbf{m i l i}^{\prime \prime}\right)\end{array}$ & $\begin{array}{c}\text { Airy } \\
\text { radius }(\mu) \\
\text { at J }\end{array}$ \\
\hline dIFS & J, H, K & 15 & 150 & 1430 & 8.6 & 1.25 & 1.7 & 19.5 \\
\hline
\end{tabular}

Table 2. Performance of the Narrow-field relay instruments.

\begin{tabular}{|c|c|c|c|c|c|c|c|c|c|c|c|}
\hline $\begin{array}{c}\text { Instrument } \\
\text { (mode) }\end{array}$ & $\lambda(\mu)$ & F/\# & FOV" & $\begin{array}{c}\text { Fld } \\
\text { curv. } \\
(\mathrm{mm})\end{array}$ & $\begin{array}{c}\text { RMS } \\
\text { WFE } \\
(\mathrm{nm})\end{array}$ & $\begin{array}{c}\text { RMS } \\
\text { Spot } \\
\text { Radius } \\
(\mu)\end{array}$ & $\begin{array}{c}\text { RMS } \\
\text { Spot } \\
\text { Radius } \\
(\text { mili-" })\end{array}$ & $\begin{array}{c}\text { Airy } \\
\text { radius } \\
(\mu)\end{array}$ & $\begin{array}{c}\text { Lateral } \\
\text { color }(\mu)\end{array}$ & $\begin{array}{c}\text { Chrom. } \\
\text { Focal } \\
\text { shift }(\mu)\end{array}$ & $\begin{array}{c}\text { Depth } \\
\text { of } \\
\text { focus* } \\
(\mathrm{mm})\end{array}$ \\
\hline $\begin{array}{c}\text { NIR im, Y- } \\
\text { band }\end{array}$ & $\begin{array}{c}0.97- \\
1.07\end{array}$ & 46 & 30 & 277 & 15.6 & 17.5 & 7.8 & 55.1 & 4 & 11.8 & 8.4 \\
\hline $\begin{array}{c}\text { NIR Im, J- } \\
\text { band }\end{array}$ & $\begin{array}{c}1.17- \\
1.33\end{array}$ & 46 & 30 & 277 & 15.6 & 18.3 & 8.2 & 66.4 & 5.5 & 17 & 10 \\
\hline $\begin{array}{c}\text { NIR Im, H- } \\
\text { band }\end{array}$ & $\begin{array}{c}1.49- \\
1.78\end{array}$ & 46 & 30 & 277 & 15.6 & 20.9 & 9.4 & 84.6 & 10.78 & 34 & 13 \\
\hline $\begin{array}{c}\text { NIR Im, K- } \\
\text { band }\end{array}$ & $\begin{array}{c}2.03- \\
2.37\end{array}$ & 46 & 30 & 277 & 15.8 & 24 & 10.8 & 115.3 & 17 & 53 & 18 \\
\hline Vis. Im, & $\begin{array}{c}0.7- \\
0.97\end{array}$ & 46 & 30 & 277 & 15.8 & 22.2 & 10.0 & 39.7 & 12.8 & 42 & 6 \\
\hline NGS/TWFS & $0.4-0.7$ & 46 & 54 & 277 & 36 & 32.3 & 14.5 & 22.7 & 0 & 0 & 3.5 \\
\hline
\end{tabular}

Table 3. Performance of the LOWFS optical relay in simultaneous $\mathrm{J}$ and $\mathrm{H}$ bands.

\begin{tabular}{|c|c|c|c|c|c|c|c|c|c|c|c|}
\hline $\begin{array}{c}\text { Instrument } \\
\text { (mode) }\end{array}$ & $\lambda(\mu)$ & F/\# & $\begin{array}{c}\text { FOV } \\
"\end{array}$ & $\begin{array}{c}\text { Fld } \\
\text { curv. } \\
(\mathbf{m m})\end{array}$ & $\begin{array}{c}\text { RMS } \\
\text { WFE } \\
(\mathbf{n m})\end{array}$ & $\begin{array}{c}\text { RMS } \\
\text { Spot } \\
\text { Radius } \\
(\mu)\end{array}$ & $\begin{array}{c}\text { RMS } \\
\text { Spot } \\
\text { Radius } \\
(\text { mili- } \\
(1)\end{array}$ & $\begin{array}{c}\text { Airy } \\
\text { radius } \\
(\mu)\end{array}$ & $\begin{array}{c}\text { Lateral } \\
\text { color } \\
(\mu)\end{array}$ & $\begin{array}{c}\text { Chrom. } \\
\begin{array}{c}\text { Focal } \\
\text { shift }(\mu)\end{array}\end{array}$ & $\begin{array}{c}\text { Depth } \\
\text { of } \\
\text { focus* } \\
(\mathbf{m m})\end{array}$ \\
\hline LOWFS & $\begin{array}{c}1.17- \\
1.78\end{array}$ & 15 & 150 & 1320 & 15.6 & 18.6 & 10.7 & 19.5 & 24.4 & 70 & 0.9 \\
\hline
\end{tabular}

Performance relaying the sodium laser guide stars into the LGSWFS is not diffraction-limited due to the fact that the sodium layer and LGS focus are not the designed conjugate points of OAP1. Of concern are the almost half-arcsecond spot sizes of the LGS WFS (see Table 4). This is a significant fraction of the 1.5 arcsecond pixels proposed for the LGS WFS. However these aberrations are of low enough order to be correctable with a single corrector such as an Alverez lens covering the entire LGS field. This corrector would vary as the distance to the sodium layer changes with telescope zenith angle.

Table 4. Performance of the LGSWFS optical system with conjugate height.

\begin{tabular}{|c|c|c|c|c|}
\hline Conjugate height & FOV, arcsec & $\begin{array}{c}\text { Field Curvature } \\
(\mathbf{m m})\end{array}$ & RMS WFE $(\mu)$ & $\begin{array}{c}\text { RMS Spot Radius } \\
(\text { milli-" })\end{array}$ \\
\hline $90 \mathrm{~km}$ & 174 & 650 & 2.8 & 415 \\
\hline $180 \mathrm{~km}$ & 162.5 & 650 & 3.1 & 440 \\
\hline
\end{tabular}

\subsection{Optical Performance 2 - Pupil Distortion}

Pupil distortion in the NGAO Cascaded relay manifests itself in at least three ways. The first is the degree to which a grid of points on the primary mirror maps to a demagnified, but square grid on the DM. Another consideration is the field dependent pupil aberrations. This effect causes the chief rays from the various field angles to not all pass through 
the center of the pupil (paraxially, the chief rays all pass through the center of the pupil, by definition, but for real rays, this is not generally true). In other words, the pupil (i.e., the DM and the correction it applies to the wavefront) is shifted with respect to the telescope primary by an amount depending on the field angle - this is similar to anisoplanatism caused by atmospheric aberrations at an altitude not conjugate to the DM. Finally, a third effect is DM-to-lenslet misregistration and scale errors.

In the analysis, the telescope primary became the "object", and field points were defined on the edges of the primary mirror. Observed field angle was set by adjusting the "stop" size placed at the Nasmyth focus to accommodate a 150 arcsecond diameter field for the woofer, and a 30 arcsecond diameter field for the tweeter in the narrow-field relay. Results are shown in Table 5.

Another factor which can degrade the performance of the AO system, and covered in Table 5, is pupil tilt on the DMs. This manifests itself not only as an uncertainty in the conjugate height of the correction, but also in noticeable plate scale errors that can degrade the astrometric accuracy. Consider a pupil re-imaged by a single parabolic mirror. That pupil image will have a curvature depending on the power of the parabola. When using a single off-axis parabola to image the pupil, one is utilizing a small part of the parent parabola's focal plane, and thus the curvature over the entire parent's focal plane appears as a tilt over the OAP's focal plane. In the case of the NGAO optical relay, this component of tilt is larger than that contributed by the incident angles on the DMs.

Table 5. Characteristics of pupil image on deformable mirrors.

\begin{tabular}{|c|c|c|c|c|c|c|c|}
\hline & $\begin{array}{c}\text { Diameter } \\
(\mathbf{m m})\end{array}$ & $\begin{array}{c}\text { Field } \\
\left({ }^{\prime \prime}\right)\end{array}$ & $\begin{array}{c}\# \\
\text { actuators }\end{array}$ & $\begin{array}{c}\text { Tilt (meters on sky, } \\
\text { peak) }\end{array}$ & $\begin{array}{c}\text { Curvature } \\
(\mathbf{m m})\end{array}$ & $\begin{array}{c}\text { Max Grid } \\
\text { Distortion }\end{array}$ & $\begin{array}{c}\text { Pupil PSF, } \\
(\mu)\end{array}$ \\
\hline $\begin{array}{c}\text { DM1, } \\
\text { woofer }\end{array}$ & $\mathbf{1 0 0}$ & $\mathbf{1 5 0}$ & $\mathbf{2 0 x 2 0}$ & $\mathbf{2 4 0}$ & $\mathbf{5 5 0 0}$ & $\mathbf{0 . 4 \%}$ & $\mathbf{4 0 0}$ \\
\hline $\begin{array}{c}\text { DM2, } \\
\text { tweeter }\end{array}$ & $\mathbf{2 5}$ & $\mathbf{3 0}$ & $\mathbf{6 4 \times 6 4}$ & $\mathbf{9 2 8}$ & $\mathbf{5 0 0}$ & $\mathbf{0 . 2 \%}$ & $\mathbf{3 8}$ \\
\hline
\end{tabular}

\section{WAVEFRONT SENSORS}

\subsection{Laser Guidestar Wavefront Sensor System}

The NGAO sensors measure wavefronts from natural and sodium guide stars to provide AO corrected images either to a 150" (science) wide field instrument or a 40" (science) narrow field instrument suite. The NGAO baseline uses 9 laser beacons that are imaged through the AO relay on to a focal plane located at the output of the first relay. Five of the nine beacons are used to generate a variable size asterism with stars on vertices of a regular pentagon with an additional central stationary beacon. NGAO also uses 3 natural stars within the 150 " FoV to provide absolute tilt and focus information. To selectively sharpen these 3 natural guide stars; we employ 3 dedicated "point-and-shoot" laser beacons that are positioned approximately 10 " outside of each of TT star radii; thus making their deployable radius 175".[6]

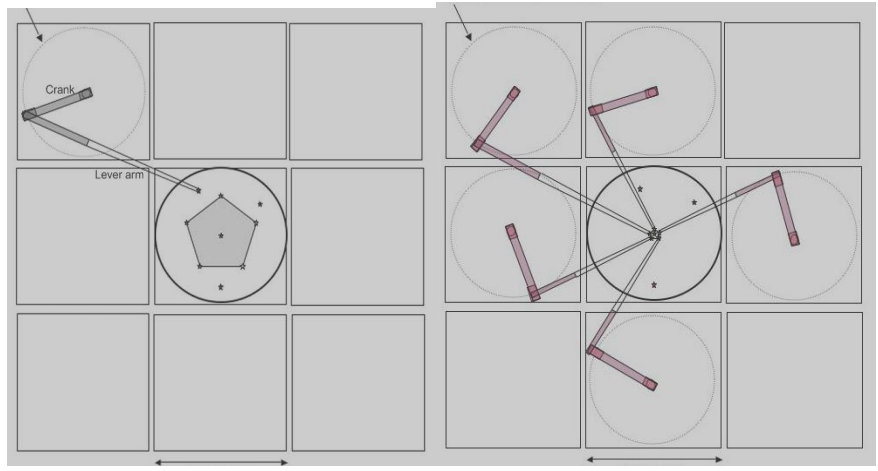

Figure 4. Plan view of the pick-off scheme showing the theta-phi mechanism. The 8 sensors that are around the central star will need to have rotation stages that ensure that the lenslet array, the woofer DM and the corresponding detector pixels stay mapped to one-another at all times. 


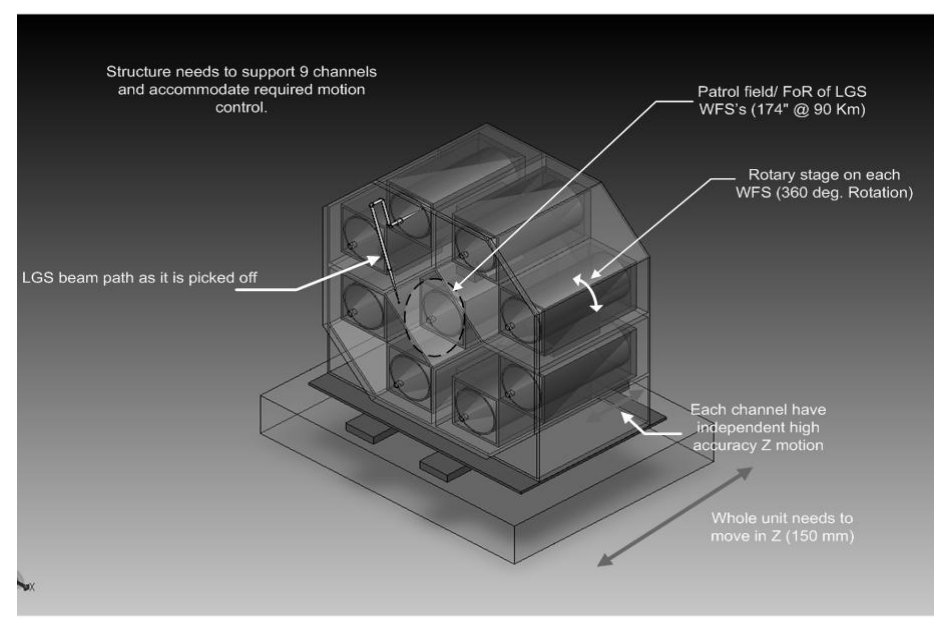

Figure 5. Schematic of the LGS WFS assembly enclosure. One of the guide star selection arms is shown.

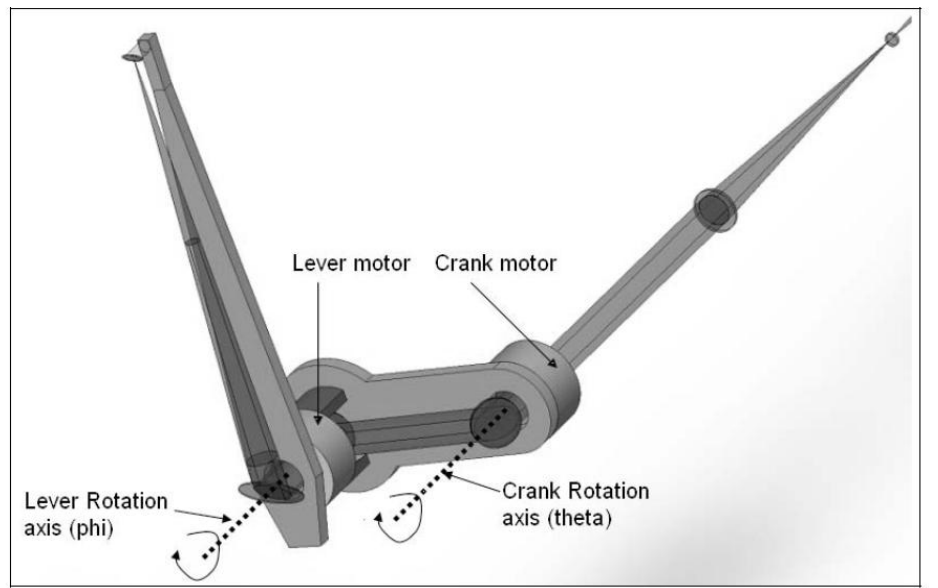

Figure 6. Theta-phi object selection mechanism with a deployable field pickoff mirror at the input and a stationary output beam feeding the wavefront sensor

Figure 7 shows a preliminary design for a $64 \times 64$ LGS wavefront sensor fed by the Keck f/13.66 beam. The Hartmann focal spots are spaced 4 pixels apart on a 256x256 detector. This geometry is in accordance with the development of JFET based low-noise fast frame readout CCD development at MIT/LL in collaboration with Keck Observatory. A 256x256 pixel rectilinear CCD detector is the nominal choice with read out speeds up to $2000 \mathrm{~Hz}$ with a 3-stage Peltier cooler. Commercial detectors with $240 \times 240$ pixels are already available, this making a graceful de-scope to a maximum of $60 \times 60$ sub-apertures a viable option. Each sensor occupies a volume of 850 x 150 x $150 \mathrm{~mm}$.

The whole LGS WFS assembly is designed to move to compensate for focal shifts with the $90-180 \mathrm{Km}$ mesospheric distance variation (LGS overall travel). To account for the curvature of the LGS focal plane with changing asterism radius, each WFS is enabled with individual de-focus of $10 \mathrm{~mm}$ with $1 \mathrm{um}$ accuracy, this high resolution stage can deal with the inaccuracies in the overall LGS assembly motion.

The orientation of the LGS field WRT to the DM rotates because of using a theta-phi pick off mechanism; a rotary stage is employed to undo this effect and keep the DM and the lenslets registered all the time. This rotation of each WFS channel is dependent on the position of the theta-phi mechanism and is envisioned as a onetime adjustment for each observation. There are 8 pick-offs that facilitate the nine WFS to guide on stars. 


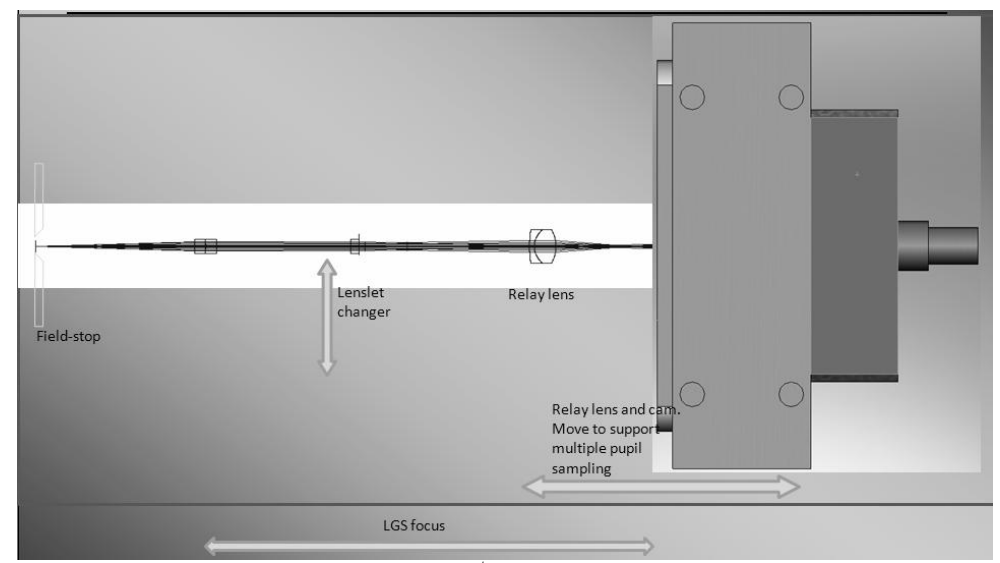

Figure 7. Schematic of a single LGS WFS channel and its $1^{\text {st }}$ order design.

\subsection{Low Order Wavefront Sensor System}

There are a total of 2 tip-tilt sensors and one TTFA sensor that are fed by the NGAO wide-field relay. The LGS WFSs and the TT(FA) sensors use the same theta-phi pickoff principle for object selection.

The TT(FA) sensors use IR detectors with variable size read-out zone. This scheme allows a wide initial capture field for the tip/tilt star since the idea is to close the AO loops first, followed by closing the tip/tilt loop on the sharpened tip/tilt star. The selectable zone size concept also allows the AO system to guide on extended objects as well as MOAO corrected point sources.

A $50 \mathrm{mas} /$ pixel plate scale has been chosen for this design based on optimizing performance by minimizing sky background. The algorithm for centroiding will start off with a larger set of pixels/sub-aperture at the start of closed loop operation and shrinks to a $2 \times 2$ pixels/subap if and when enough correction is achieved. The level of shrinkage will depend on the amount of correction and the size of the object.

Laboratory tests have demonstrated that Hawaii 2RG infrared arrays can be read out fast over small subregions and obtain low enough levels of read noise to be suitable for fast tip/tilt measurements. [9]

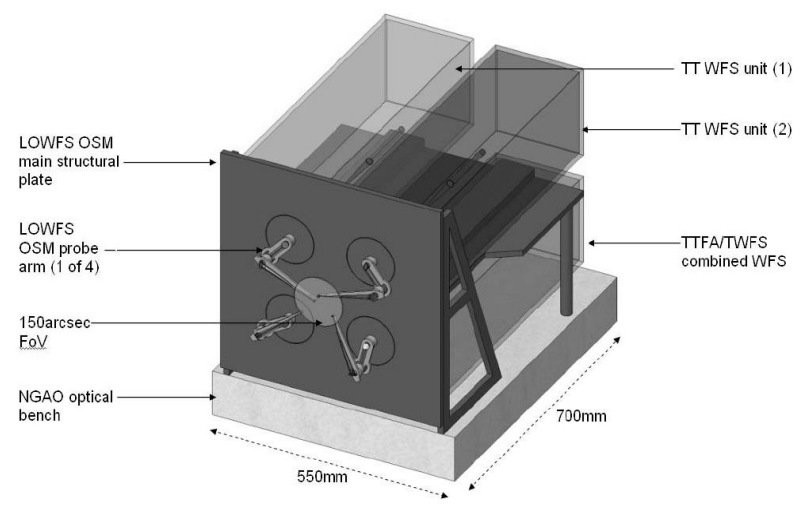

Figure 8. LOWFS assembly showing four pick off arms. One arm feeds the TTFA/ TWFS assembly, 2 more arms feed the

TT sensors and one arm feeds a PSF monitor (not part of the baseline design, so this is a place holder)

\subsection{Natural Guidestar Wavefront Sensor}

The NGS sensor will be used when bright guidestars can be used for atmospheric wavefront sensing in place of the lasers. The sensor will be positioned at the output of the narrow field relay, equipped to pick off stars using field steering mirrors / dichroics over a 40 arcsec field of regard. Since the TWFS and the NGS sensor will not be used simultaneously, thy can share the same articulated dichoric as the first field steering mirror. But the NGS WFS and the TWFS will use separate cameras, instead of the TWFS being a role played by a $5 \times 5$ lenslet option of the NGS HOWFS, for the following reasons: 
1) NGS sensors are built for high speed readout (low readout noise but significant dark current). Even though they are cooled to $-20 \mathrm{deg}$. C, dark current will likely be a limiting factorr for these sensors are used in long exposures needed for TWFS.

2) The current generation of high speed (e.g. SciMeasure) camera control architectures implement a CDS method of reading out detectors using a clamp and filter based circuit. This circuit is optimized to work with a finite number of programs (gains, speed with optimized filter and clamp settings) for speeds between 25 and $2000 \mathrm{~Hz}$. The TWFS will typically need to run very slowly, $0.01-10 \mathrm{~Hz}$ and will need optimized circuitry for that particular application.

\subsection{Truth Wavefront Sensor}

A Truth or Calibration Wavefront Sensor is used to calibrate biases that arise when using laser guide stars. The biases are principally caused by the elongated nature of the LGS when viewed by sub-apertures of the laser guide star wavefront sensor and the changing sodium layer density profile. The truth wavefront sensor measures these biases by sensing the wavefront from a point source (a natural star). These biases are slowly varying and are of a low spatial order, so, a natural guide stars WFS using long exposures and only measuring the lowest spatial wavefront error is sufficient.

The nominal design for the NGAO truth sensor is a $5 \times 5$ subaperture Hartmann sensor sensing visible light from a natural star in the narrow field relay. A TWFS in this position can be used to precisely calibrate the on-axis wavefront entering the narrow field high-resolution science instruments. Because this star is partially sharpening by AO in this field, current estimates suggest that the visible TWFS can go down to 21.5 mag with $10 \mathrm{sec}$. integration time and achieve $35 \mathrm{~nm}$ of total TWFS error.

Some consideration has been given to designing a truth sensor for calibrating wavefronts on the output of the wide field relay as well. This sensor might either use light split from the natural star entering the TTFA sensor. This light will be sharpened by a MEMS DM operating with MOAO correction, enabling dim stars to be useful.

\section{TOMOGRAPHIC PROCESSING AND CONTROL}

\subsection{Real Time Controller}

The Real Time Controller (RTC) is a specialized computer system designed to perform all of the wavefront sensing, tomography calculations, and deformable mirror control processing at rates that keep up with atmospheric turbulence induced optical aberrations. The RTC data flow and computer architectures have been designed to achieve the tomography precision, noise suppression, and bandwidth requirements implied by the science-case driven wavefront error budgets.

An equally important consideration in the RTC design is the need to keep the cost and complexity manageable. Simply scaling earlier implementations of single conjugate AO RTC reconstructors using traditional central processing units (CPUs) is infeasible because of the multiplying effect of multi-guidestars and multiple deformable mirrors on computer speed requirements. To address this issue, we have taken advantage of the parallelizability of wavefront reconstruction and tomography algorithms and mapped them on to a massively-parallel processing (MPP) compute architecture. This architecture scales in size and complexity much more favorably than doing the same calculations on CPUs, and can be readily implemented with commercial off the shelf technology building blocks called field programmable gate arrays (FPGAs).[10]

The RTC, at the core, performs the following tasks in laser guidestar (LGS) mode:

- Accept digitized pixel data from the Hartmann and tip/tilt sensor cameras, and perform basic image processing (dark and bias subtraction, flat-fielding).

- Calculate wavefront tilts from the Hartmann and tip/tilt data using a choice of noise-optimal centroiding algorithms, and compensate for non-linearities in the centroiding methods.

- Reconstruct wavefront phase at each sensor and transmit this information to the tomography processor

- Use cone-beam projection tomography techniques to reconstruct the three-dimensional volume of atmospheric index-of-refraction variations above the telescope and over the field of view of interest, given the wavefronts from various directions as measured by each wavefront sensor. This algorithm also taske inputs from the tip/tilt and tip/tilt/focus/astigmatism sensors to break volume solution ambiguities inherent in laser wavefront measurements. 
- Integrate through the volume of index estimates along paths to the science instruments - either on-axis for the narrow field science modes, or along the different directions for the deployable integral field spectrograph arms and tip/tilt/focus sensors.

- Compensate for deformable mirror influence functions and nonlinearities, deriving voltage commands for the DMs given desired wavefront distortion corrections to be placed on them. Split the control between commands to the tip/tilt mirror, low-order (woofer) DM, and high order (tweeter) DMs.

- Incorporate control compensation dynamics to keep the system stable and achieving a given temporal bandwidth.

\subsection{Non Real Time Controller}

An overall supervisory control system maintains interactions with the telescope, AO operator, low bandwidth devices on the AO bench, devices in the laser launch system, the laser control system, the laser safety system, the diagnostic data archiving system, and the $\mathrm{AO}$ real time controller. The software is organized into a hierarchy, coordinated from above by science operations tools. The software includes an AO sequencer, motion (motor) control, device (cameras, environmental, power) control, RTC control, laser sequencer, laser motion control, laser device, environment, and safety control, and an archival data server. All the critical safety control elements are independent hard-wired interlock systems, with the supervisory control having authority to monitor or shut down systems.

\section{NARROW FIELD INSTRUMENT PATHS}

The second relay in the NGAO system is designed to feed a number of narrow field science instruments including:

- Narrow field infrared integral field spectrometer

- Narrow field infrared imager

- Narrow field visible imager

In addition, the narrow field relay will provide light to the natural guide star and LGS mode truth wavefront sensor.

As a temporary measure, NGAO will be designed to feed the existing OSIRIS instrument. It will also feed the Keck Interferometer dual star module and the 'OHANA interferometer.

Observing efficiency requirements dictated that NGAO be quickly reconfigurable to feed each of these instruments without having to physically move an instrument into place on output ports. The "optical switchyard" arrangement shown in Figure 9 shows a feasible layout that serves instruments simply by changing splitter configurations. In order to fit all the beam lines and splitters, the output $\mathrm{f} / \#$ of the second relay needs to be on the order of $\mathrm{f} / 48$, rather than matching the input $\mathrm{f} / \#$ of $\mathrm{f} / 13$.

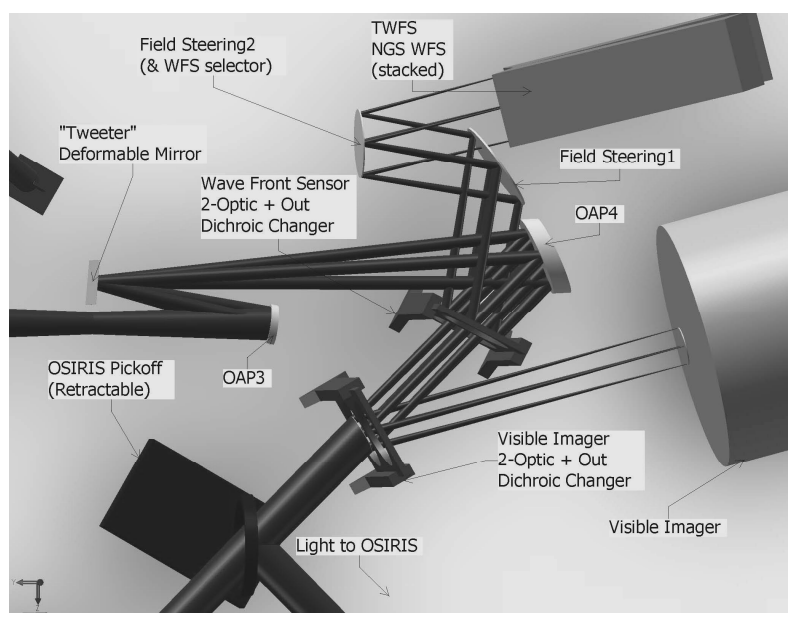

Figure 9. Narrow Field Path Output detail. Note that it appears that Field Steering1 mirror vignettes the light traveling to the wave front sensors. This is however, the available field for natural guide stars - not the field required for the wave front sensors. The wave front sensors accept a much smaller field. 


\subsection{Science Observing Modes}

Table 6 below lists the various configuration setups vs science observing mode for Keck NGAO, highlighting the fact that the NGAO system design is a reconfigurable and multi-functional support system for various diffraction-limited science observing needs.

\section{CONCLUSION}

The Keck NGAO design underwent a System Design Review (basically the equivalent of a Feasibility Design Review) in April 2008 and is now entering Preliminary Design Phase. The initial design exercise has resulted in a proposed AO architecture that supports a variety of AO science cases. The system employs closed-loop control of a woofer DM and MOAO open-loop control of several MEMS DMs, one being 64 actuators across for narrow field high-resolution science, and several others being 32 across for the arms of deployable integral field spectrometers on a wide field. A distinguishing feature of this $\mathrm{AO}$ system will be the use of tomographic wavefront sensing with multiple laser guide stars and the use of MOAO to sharpen tip/tilt stars to increase LGS sky coverage.

Table 6. NGAO Science Observing Modes

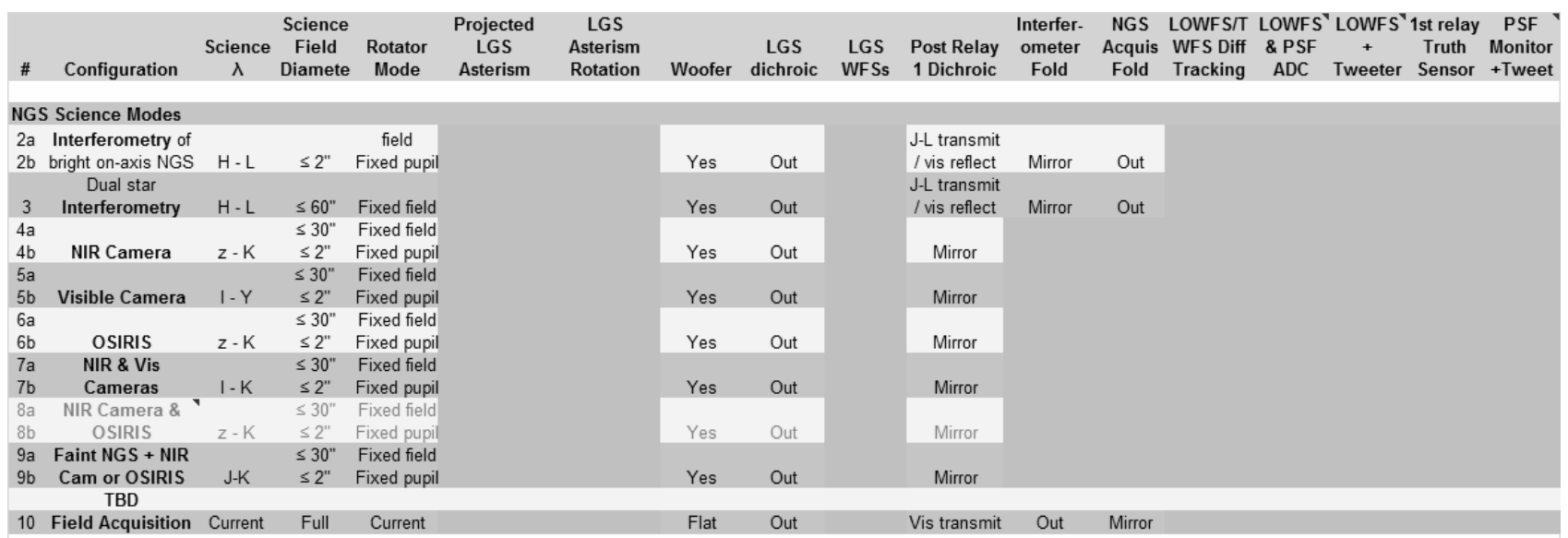

\begin{tabular}{|c|c|c|c|c|c|c|c|c|c|c|c|c|c|c|c|c|c|}
\hline \multicolumn{18}{|c|}{ LGS Science Modes } \\
\hline 1 & d-IFS & $z-K$ & $\leq 120^{\prime \prime}$ & Fixed field & Wide & Yes for field & Yes & $\ln$ & 9 & Out & \multirow{2}{*}{$\begin{array}{c}\text { Out } \\
\text { J transmit / } \\
\text { HK reflect }\end{array}$} & \multirow{2}{*}{$\begin{array}{l}\text { Out } \\
\text { Out } \\
\text { Out }\end{array}$} & \multirow{2}{*}{$\begin{array}{l}\text { No } \\
\text { No }\end{array}$} & \multirow{2}{*}{$\begin{array}{l}\text { Tracking } \\
\text { Tracking }\end{array}$} & 3 & \multirow{2}{*}{$\begin{array}{l}\text { Yes } \\
\text { Yes, on- } \\
\text { axis }\end{array}$} & \multirow{2}{*}{$\begin{array}{c}\text { Option } \\
\text { Option } \\
\text { Unlikely }\end{array}$} \\
\hline $\begin{array}{l}2 a \\
2 b\end{array}$ & $\begin{array}{l}\text { Interferometry of } \\
\text { faint on-axis NGS }\end{array}$ & $H-L$ & $\leq 2^{\prime \prime}$ & $\begin{array}{c}\text { Fixed } \\
\text { Fixed pupil }\end{array}$ & Narrow & $\begin{array}{l}\text { Yes for field } \\
\text { No, fixed }\end{array}$ & Yes & $\ln$ & 6 & Out & & & & & 1 on-axis & & \\
\hline 3 & $\begin{array}{c}\text { Dual star } \\
\text { Interferometry }\end{array}$ & $\mathrm{H}-\mathrm{L}$ & $\leq 60^{\prime \prime}$ & Fixed field & $\leq$ Medium & Yes for field & Yes & $\ln$ & 9 & Out & $\begin{array}{l}\text { J transmit / } \\
\text { HK reflect }\end{array}$ & Out & No & Tracking & 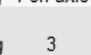 & Yes & Option \\
\hline $4 a$ & & & $\leq 30^{\prime \prime}$ & Fixed & $\leq$ Medium & Yes for field & & & 9 & $\mathrm{JH}$ transmit & & & Option & & 3 & Option & Option \\
\hline $4 \mathrm{~b}$ & & $\mathrm{~K}$ & $\leq 2^{\prime \prime}$ & Fixed pupil & Narrow & No, fixed & & & 6 & $/ \mathrm{K}$ reflect & & & No & & 1 on-axis & Unlikely & Unlikely \\
\hline $4 \mathrm{c}$ & & & $\leq 30^{\prime \prime}$ & Fixed & $\leq$ Medium & Yes for field & & & 9 & $\mathrm{~J}$ transmit / & & & Option & & 3 & Option & Option \\
\hline $4 d$ & & $\mathrm{H}$ & $\leq 2 "$ & Fixed pupil & Narrow & No, fixed & & & 6 & $\mathrm{H}$ reflect & & & No & & 1 on-axis & Unlikely & Unlikely \\
\hline $4 e$ & & & $\leq 30^{\prime \prime}$ & Fixed & $\leq$ Medium & Yes for field & & & 9 & $\mathrm{H}$ transmit & & & Option & & 3 & Option & Option \\
\hline $5 a$ & & & $\leq 30^{\prime \prime}$ & Fixed field & $\leq$ Medium & Yes for field & & & 9 & $\mathrm{JH}$ transmit & & & Option & & 3 & Option & Option \\
\hline $5 b$ & Visible Camera & $0.7-1 \mu \mathrm{m}$ & $\leq 2^{\prime \prime}$ & Fixed pupil & Narrow & No, fixed & Yes & In & 6 & / vis reflect & Out & Out & No & Tracking & 1 on-axis & Unlikely & Unlikely \\
\hline $6 a$ & & & $\leq 30^{\prime \prime}$ & field & $\leq$ Medium & Yes for field & & & 9 & $\mathrm{JH}$ transmit & & & Option & & 3 & Option & Option \\
\hline $6 \mathrm{~b}$ & & $\mathrm{~K}$ & $\leq 2 "$ & Fixed pupil & Narrow & No, fixed & & & 6 & $/ \mathrm{K}$ reflect & & & No & & 1 on-axis & Unlikely & Unlikely \\
\hline $6 c$ & & & $\leq 30^{\prime \prime}$ & Fixed & $\leq$ Medium & Yes for field & & & 9 & $\mathrm{~J}$ transmit / & & & Option & & 3 & Option & Option \\
\hline $6 \mathrm{~d}$ & & $\mathrm{H}$ & $\leq 2^{\prime \prime}$ & Fixed pupil & Narrow & No, fixed & & & 6 & $\mathrm{H}$ reflect & & & No & & 1 on-axis & Unlikely & Unlikely \\
\hline $6 \mathrm{e}$ & & & $\leq 30^{\prime \prime}$ & Fixed & $\leq$ Medium & Yes for field & & & 9 & $\mathrm{H}$ transmit & & & Option & & 3 & Option & Option \\
\hline $6 f$ & OSIRIS & $z-J$ & $\leq 2^{\prime \prime}$ & Fixed pupil & Narrow & No, fixed & Yes & In & 6 & /zJ refl & Out & Out & No & Tracking & 1 on-axis & Unlikely & Unlikely \\
\hline $7 a$ & & & $\leq 30^{\prime \prime}$ & Fixed field & s Medium & Yes for field & & & 9 & $\mathrm{JH}$ transmit & & & Option & & 3 & Option & Option \\
\hline $7 \mathrm{~b}$ & & I.K & $\leq 2$ & Fixed pupil & Narrow & No, fixed & Yes & In & 6 & / IK reflect & Out & Out & No & Tracking & 1 on-axis & Unlikely & Unlikely \\
\hline $7 \mathrm{e}$ & NIR \& Vis & & $\leq 30^{\prime \prime}$ & Fixed field & $\leq$ Medium & Yes for field & & & 9 & $\mathrm{H}$ transmit / & & & Option & & 3 & Option & Option \\
\hline $7 f$ & Cameras & $\mathrm{I}, \mathrm{J}$ & $\leq 2$ & Fixed pupil & Narrow & No, fixed & Yes & In & 6 & IJ reflect & Out & Out & No & Tracking & 1 on-axis & Snlikely & Unlikely \\
\hline $8 a$ & NIR Camera \& & & $\leq 30^{\prime \prime}$ & Fixed field & $\leq$ Medium & Yes for field & & & 9 & Same as & & & No & & 3 & Option & Option \\
\hline $8 b$ & OSIRIS & $z-K$ & $\leq 2^{\prime \prime}$ & Fixed pupil & Narrow & No fixed & Yes & $\ln$ & 6 & NIR Cam & Out & Out & No & Tracking & 1 on-axis & Snlikely & Unlikely \\
\hline & TBD & & & & & & & & & & & & & & & & \\
\hline 10 & Field Acquisition & Current & Full & Current & & & Flat & In & & Vis transmit & Current & Mirror & Current & Current & Current & Current & Current \\
\hline 11 & LGS Acquisition & Current & Full & Current & Current & Current & Flat & In & Current & Current & Current & & & & & & \\
\hline
\end{tabular}




\section{ACKNOWLEDGEMENTS}

The W. M. Keck Observatory is operated as a scientific partnership among the California Institute of Technology, the University of California, and the National Aeronautics and Space Administration. The Observatory was made possible by the generous financial support of the W. M. Keck Foundation.

\section{REFERENCES}

[1] Peter Wizinowich, David Le Mignant, Antonin Bouchez, 1 Randy Campbell, Jason Chin, Adam Contos, 2 Marcos van Dam, Scott Hartman, Erik Johansson, Robert Lafon, Hilton Lewis, Paul Stomski, and Douglas Summers, "The W. M. Keck Observatory Laser Guide Star Adaptive Optics System: Overview," Publications of the Astronomical Society of the Pacific, 118:297-309 (2006). See also a Bibliography of Keck AO Science Papers at http://www2.keck.hawaii.edu/library/biblios/aokeck.php.

[2] P. Wizinowich, R. Dekany, D. Gavel, C. Max, S. Adkins, B. Bauman, J. Bell, A. Bouchez, M. Britton, J. Chin, R. Flicker, E. Johansson, R. Kupke, D. Le Mignant, C. Lockwood, D. Medeiros, E. McGrath, A. Moore, C. Neyman, M. Reinig, V. Velur, "W. M. Keck Observatory's next-generation adaptive optics facility,” SPIE 7015 (2008).

[3] Claire Max, Elizabeth McGrath, Donald Gavel, Richard Dekany, Peter Wizinowich, David Le Mignant, "The science case for the next-generation AO system at W. M. Keck Observatory," SPIE 7015 (2008).

[4] David Le Mignant, Elizabeth McGrath, "W. M. Keck Observatory's next-generation adaptive optics facility: science operations," SPIE 7015 (2008).

[5] Bruce Macintosh, James Graham, David Palmer, René Doyon, Jennifer Dunn, Donald Gavel, James Larkin, Ben Oppenheimer, Les Saddlemyer, Anand Sivaramakrishnan, J. Kent Wallace, Brian Bauman, Darren Erickson, Christian Marois, Lisa Poyneer, Remi Soummer, "The Gemini planet imager: from science to design to construction," SPIE 7015 (2008).

[6] Richard Dekany, Ralf Flicker, Christopher Neyman, "Sharpening low-order NGS using patrolling laser guide stars," SPIE 7015 (2008).

[7] Morzinski, Katie M.; Gavel, Donald T.; Norton, Andrew P.; Dillon, Daren R.; Reinig, Marco R., "Characterizing MEMS deformable mirrors for open-loop operation: high-resolution measurements of thin-plate behavior," SPIE, 6888, 68880S-68880S-12 (2008).

[8] Donald Gavel, Brian Bauman, Marco Reinig, Christopher Lockwood, Kathleen Morzinski, Scott A. Severson, David W. Palmer, Daren Dillon, Mark Ammons, Bryant Grigsby, Elinor Gates, Jess Johnson, "Visible light laser guidestar experimental system (Villages): on-sky tests of new technologies for visible wavelength all-sky coverage adaptive optics systems," SPIE 7015 (2008).

[9] Viswa Velur, Ralf Flicker, Richard Dekany, Gustavo Rahmer, Roger Smith, "Low-order wavefront sensing in tomographic multibeacon adaptive optics systems,” SPIE 7015 (2008).

[10] Gavel, Donald; Reinig, Marc; Cabrera, Carlos, "Fast hardware implementation of tomography for multi-guidestar adaptive optics," SPIE 5903, 138-147 (2005). 